\title{
Источники питания TDK-Lambda серии GXE: управляемая мощность в промышленном конструктиве
}

\author{
Е. Рабинович ${ }^{1}$
}

УДК 621.311.6 | ВАК 05.11.01

\begin{abstract}
Компания TDK-Lambda представила новую серию источников питания с широким набором функций для различных областей применения. Притом что устройства серии GXE предлагаются в типично промышленном исполнении, главная их особенность - возможность работы как в стандартном режиме стабилизации напряжения, так и в режиме стабилизации выходного тока. Благодаря наличию нескольких режимов управления устройства наделены возможностями программируемого источника питания даже в отсутствие элементов управления на передней панели. Рассмотрим особенности конструкции, режимы работы, коммуникационные возможности и области применения новых приборов.
\end{abstract}

K онструкция источников питания серии GXE представляет собой печатную плату, закрепленную на металлической платформе U-образного профиля (рис. 1). Прибор предусматривает безвентиляторное охлаждение: платформа и несколько радиаторов выполняют функцию теплоотвода. Охлаждение внешним потоком воздуха позволяет расширить возможности и улучшить характеристики источника питания. Так, например, в диапазоне входного напряжения от 85 до 170 В при потоке воздуха всего 1 м / с мощность устройства не снижается. Модуль размерами $41 \times 127 \times 254$ мм подходит для размещения в стандартных корпусах высотой $1 \bigcup$ и обеспечивает выходную мощность 600 Вт. Заказчикам доступно также исполнение с верхней крышкой, когда требуется внешний монтаж источника питания. В серии GXE предлагаются две модификации устройства - с входными номинальными напряжениями 24 и 48 В постоянного тока

Отличие изделия от стандартного источника питания состоит в нескольких режимах работы и гибкости настроек, что, в свою очередь, обусловлено наличием как аналогового, так и цифрового портов управления, доступ к ним осуществляется через 20-пиновый вертикальный коннектор управления производства компании JST (рис. 2). все выводы коннектора

Компания TDK-Lambda, инженер технической поддержки, Evgeny.R@tdk-lambda.ru. управления обозначены на блок-схеме источника питания (см. рис. 5).

Аналоговый порт включает в себя сигналы дистанционного включения-выключения (выводы CNT7-CNT2 на рис. 5), сигнал рабочего состояния выхода Power Fail (вывод PF на рис. 5), сигнал наличия нормированного входного напряжения AC Fail и выводы дистанционной обратной связи +S/-S. Через аналоговый порт также обеспечивается регулировка выходного напряжения в диапазоне 20-120\% и токоограничения в диапазоне 20-115\% от номинальных значений напряжения и тока. Данные установки задаются строго по пропорционально-линейному закону с помощью сигнала постоянного напряжения в диапазоне 0-6 В через выводы PV и СС. Для более простого и гибкого интегрирования устройства в различные системы инженеры могут воспользоваться выводами +STB /-STB источника вспомогательного резервного питания номиналом 5 В и выводом РС распределения тока нагрузки для параллельного включения нескольких модулей питания и увеличения выходной мощности.
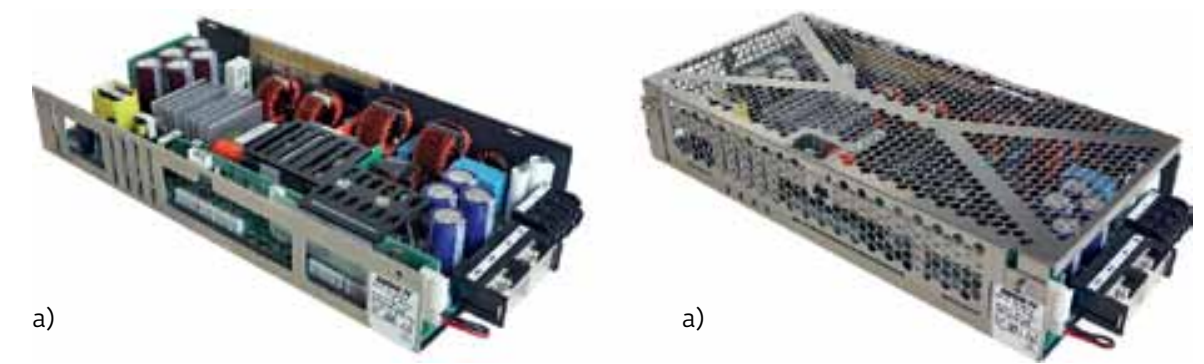

Рис. 1. Источник питания серии CXE600: а - стандартное исполнение; 6 - исполнение с крышкой 


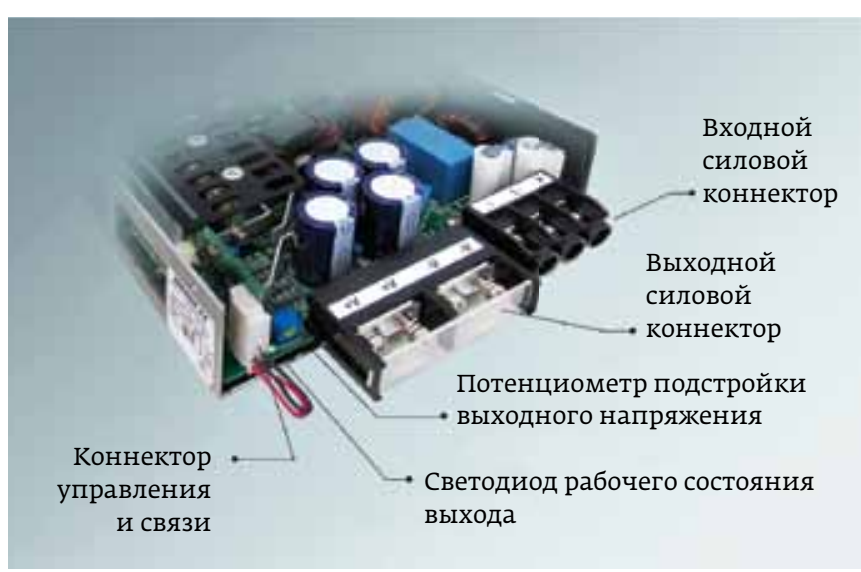

Рис. 2. Органы управления и индикации источника питания серии GXE600

Цифровая коммуникация с источником питания производится по протоколу Modbus RTU на базе интерфейса RS-485, что значительно расширяет возможности связи по сравнению с интерфейсом I², часто используемым для AC / DC-источников. Реализованный через тот же сигнальный коннектор цифровой порт управления не только позволяет устанавливать выходные параметры, но и обеспечивает возможность работы с целым рядом дополнительных параметров, часто используемых на практике. Например, доступны команды регулировки длительности фронта нарастания, считывания рабочей температуры, настройки уровня защиты, считывания времени наработки прибора и даже остаточного расчетного ресурса электролитических конденсаторов.

Управлять прибором можно не только через специальный Modbus-контроллер, но и через стандартный компьютерный USB-порт и специальную программную платформу GXE aXess с графическим интерфейсом для удобства отладки пользователями. Данная графическая оболочка, устанавливаемая на OC Windows,

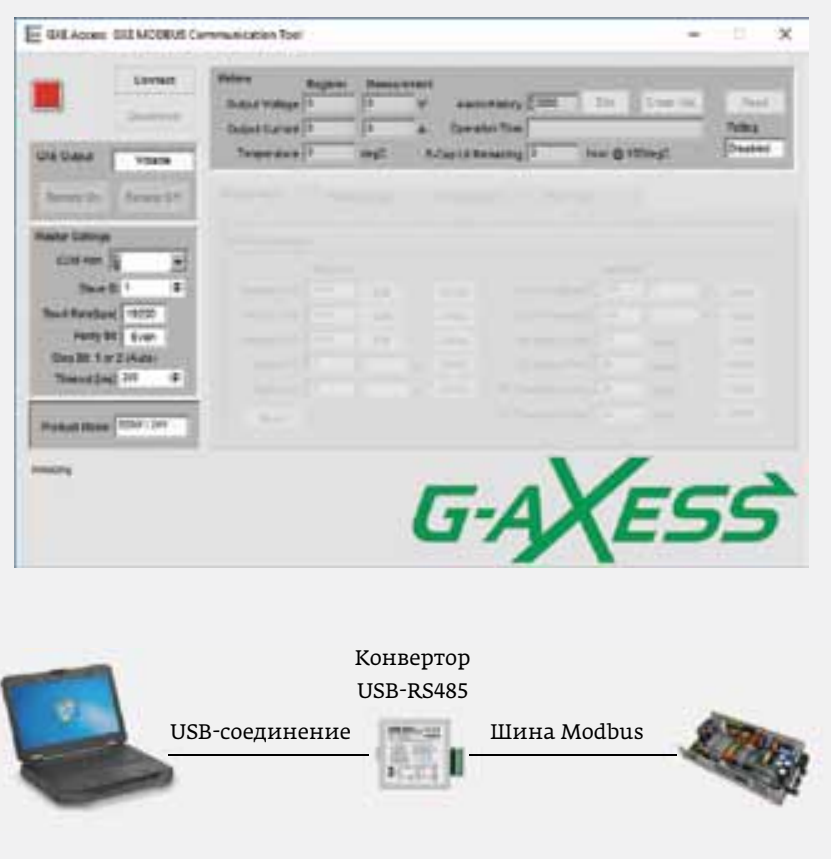

Рис. 3. Подсоединение ИП серии СХЕ600 к хосту-компьютеру и вид основного окна приложения GXE aXess

позволяет осуществлять настройки в привычном десятичном виде и автоматически преобразовывать данные в формат протокола. Подсоединение к источнику питания обеспечивается через преобразователь USB-RS485, поддерживающий полудуплексный режим связи и автоматический обмен данными по шине RS485 (рис. 3). Скорость обмена данными составляет от 2400 до 19200 бод, а рекомендуемая длина коммуникационной шины - до 100 м.

Возможно подсоединение нескольких блоков серии GXE к цифровой шине с рекомендуемым количеством модулей-приемников до 15 шт. (рис. 4). При множественном подсоединении необходимо заранее установить для

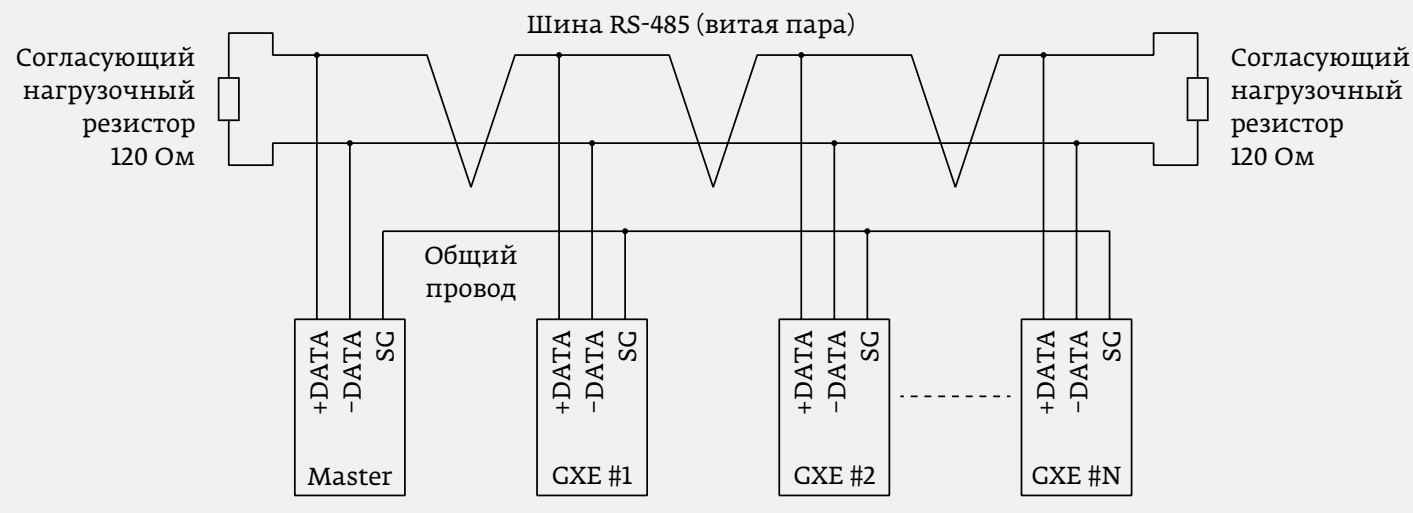

Рис. 4.

Подсоединение нескольких ИП серии GXE600 к цифровой шине 
каждого прибора свой уникальный адрес (slave ID). Интерфейсная шина состоит из двух линий (+DATA/-DATA), подключаемых к соответствующим выводам объединяемых приборов, и общего заземляющего провода (вывод SG), подключение которого не обязательно, но зачастую целесообразно для обеспечения стабильной коммуникации.
Наряду с общим руководством по эксплуатации для пользователей доступны руководство по применению приложения GXE aXess (GXE GUI guide) и отдельное руководство по коммуникации (Communication Manual), в котором подробно описан протокол, используемый формат данных и порядок работы с каждым из внутренних регистров обмена данными.

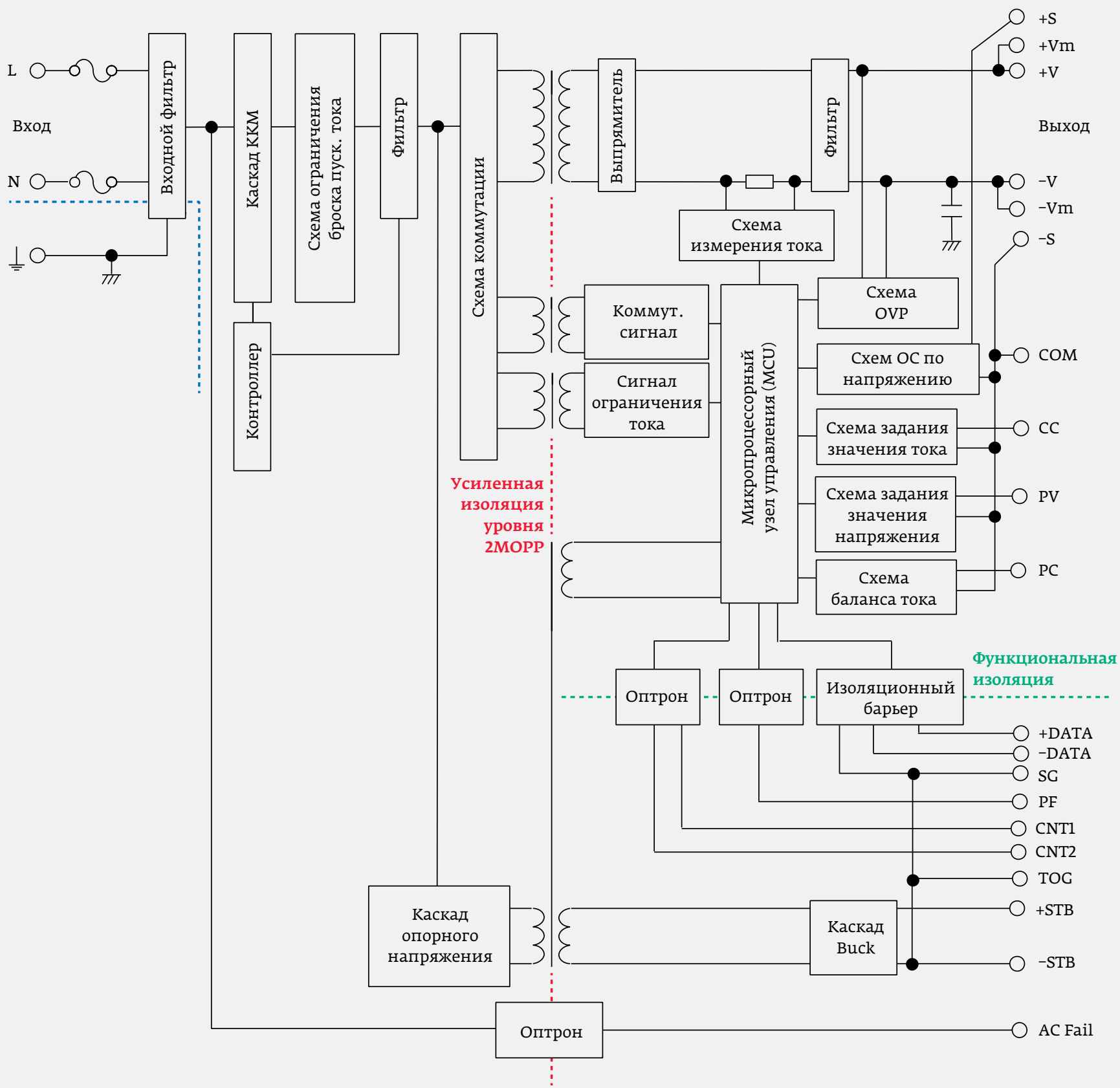

Рис. 5. Блок-схема иП серии СXЕ600 
Что касается выбора режима работы, то при стандартных настройках (когда нет дополнительных установок по токоограничению по аналоговому или цифровому порту) источник питания функционирует в режиме стабилизации выходного напряжения во всем диапазоне токов вплоть до значения выходного тока, равного 1,15 от номинального, при котором схема переходит в режим ограничения по току в режиме Ніссир. Если определенная установка по току (CC reference) задана, устройство переводится в режим стабилизации тока по всей допустимой шкале напряжения вплоть до значений программной установки по напряжению (CV reference) либо аппаратного порога по перенапряжению (HWOVP Hardware Over Voltage Protection). Блок-схема источника питания серии GXEGO0 показана на рис. 5.

Таким образом, GXEG00 действительно гибкое устройство. Например, подстройка напряжения возможна с помощью встроенного потенциометра в диапазоне + /-20\% от номинала, через аналоговый порт управления в диапазоне 20-120\%, а также через цифровой порт управления в том же диапазоне. Для защиты от перенапряжения предусмотрено не только схемотехническое решение с фиксированным порогом срабатывания, но и подстраиваемая схема, допускающая программное изменение точки срабатывания.
По согласованию с производителем пользователи могут заказывать модели в опциональном исполнении, в частности с верхней крышкой, без дистанционного управления, для жестких условий эксплуатации, для работы от сети постоянного тока и др.

Изделия серии GXE штатно работают в диапазоне температур от -20 до $70{ }^{\circ} \mathrm{C}$ со снижением мощности при температуре выше $50^{\circ} \mathrm{C}$ в соответствии с графиками, приведенными в спецификации. При этом запуск источника питания гарантирован при температуре от $-40^{\circ} \mathrm{C}$. Стоит также отметить высокую эффективность изделий - КПД модулей в зависимости от ряда внешних параметров может достигать 95\% при входном напряжении 220 В и полной нагрузке. При этом поддерживаемый семилетний гарантийный период по достоинству могут оценить даже самые требовательные заказчики.

Серия GXE отвечает требованиям стандартов CSA/EN/UL 60950-1, сертифицирована на соответствие стандарту EN60601-1 для приборов медицинского назначения. Это позволяет успешно применять устройства в широком спектре приложений: в системах измерений и выходного контроля, промышленном оборудовании, освещении, системах связи, медицинском оборудовании и прочих системах. 